\title{
FACTORES ASOCIADOS AL EMBARAZO ADOLESCENTE EN UN CENTRO MATERNO INFANTIL DE LIMA, PERÚ
}

\author{
FACTORS ASSOCIATED WITH ADOLESCENT PREGNANCY IN A MATERNAL AND CHILD CENTER IN \\ LIMA, PERU
}

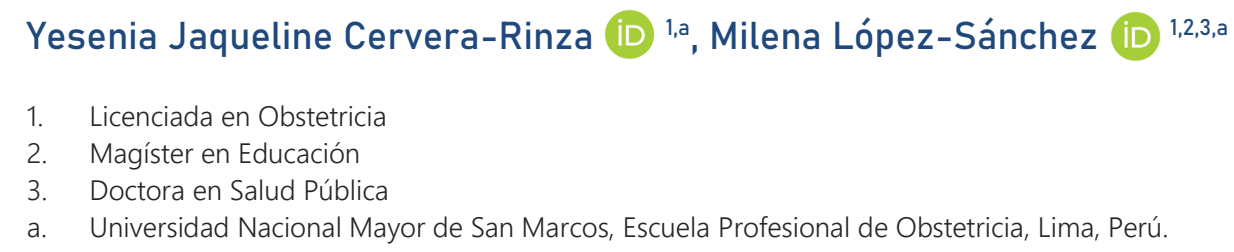

\section{RESUMEN}

Objetivo: Determinar los factores asociados al embarazo adolescente en un centro materno infantil de Lima durante el 2018. Material y métodos: Estudio analítico de casos y controles. Se obtuvo muestra de 57 adolescentes atendidas en el Centro de Salud Materno Infantil "Santa Anita", divididas en 19 adolescentes con embarazo (casos) y 38 adolescentes sin embarazo (controles). La técnica fue la encuesta mediante cuestionario, previo consentimiento informado. El análisis se realizó en el software estadístico STATA. La asociación se evaluó mediante la prueba Chi Cuadrado de Pearson, mientras que la diferencia de medias se analizó mediante T de Student. Resultados: Dentro de los factores individuales, se halló asociación significativa entre el embarazo adolescente con: el estado civil $(p<0.001)$, grado de instrucción $(p=0.005)$, ocupación ( $p 00.002)$, uso de métodos anticonceptivos $(p=0.008)$, edad de las adolescentes $(p<0.001)$, edad de la primera relación sexual $(p=0.039)$ y andría $(p<0.001)$. En los factores familiares, se demostró asociación significativa con: el grado de instrucción de los padres $(p=0.002)$ y el hecho de que algún miembro de la familia haya tenido un embarazo adolescente $(p=0.021)$. En los factores sociales, se demostró asociación significativa con el número de personas que viven en el hogar $(p=0.012)$. Conclusión: Los principales factores asociados al embarazo adolescente fueron el estado civil, la edad del adolescente, la andría y el grado de instrucción de los padres.

Palabras clave: Factores de riesgo, Embarazo adolescente, Salud del adolescente, Prevención primaria (Fuente: DeCS, BIREME)

Financiamiento

Autofinanciado

Conflicto de interés

Las autoras declaran no tener conflicto de interés

Proceso editorial

Recibido: 13 de Enero del 2020

Aprobado: 20 de Marzo del 2020

Correspondencia

Yesenia Cervera

yesenia.jcr@hotmail.com

\section{Cita bibliográfica}

Cervera-Rinza YJ, López-Sánchez M Factores asociados al embarazo adolescente en un centro materno infantil de Lima Revista Internacional de Salud Materno Fetal. 2020; 5(1): 36-42.

\section{Abstract}

Objective: To determine the factors associated with adolescent pregnancy in a maternal and child center in Lima during 2018. Material and methods: Analytical case and control study. A sample of 57 adolescents was obtained at the Maternal and Child Health Center "Santa Anita", divided into 19 adolescents with pregnancy (cases) and 38 adolescents without pregnancy (controls). The technique was the questionnaire survey, with prior informed consent The analysis was performed in the statistical software STATA. The association was evaluated using Pearson's Chi Square test, while the difference in media was analyzed by Student's Ttest. Results: Within the individual factors, association between adolescent pregnancy with marital status $(p<0.001)$, degree of instruction $(p=0.005)$, occupation $(p=0.002)$, use of contraceptive methods $(p=0.008)$, age of adolescents $(p<0.001)$, age of first intercourse $(p=0.039)$ and andria $(p<0.001)$. In the family factors, a significant association was found with the degree of instruction of the parents $(p=0.002)$ and the fact that some member of the family had an adolescent pregnancy $(p=0.021)$. In social factors, a significant association was shown with the number of people living in the home $(p=0.012)$. Conclusion: The main factors associated with pregnancy were the marital status, the age of the adolescent, the andria and the degree of instruction of the parents.

Keywords: Risk factors, Pregnancy in adolescence, Adolescent health, Primary prevention (Source: MeSH, NLM) 


\section{INTRODUCCIÓN}

El embarazo adolescente es una problemática de salud pública que, a pesar de las intervenciones realizadas, no ha logrado disminuir sus indicadores, siendo Latinoamérica la región con menor progreso en esta tarea', la cual presenta una escasa educación en planificación familiar y bajo compromiso social en la difusión educativa. ${ }^{2}$ En el Perú, durante el 2017, un $13.4 \%$ de las adolescentes entre 15 a 19 años fueron madres 0 quedaron embarazadas por primera vez, cifra que incrementó con respecto al año previo donde se obtuvo un $12.7 \%{ }^{3}$

La preocupación del embarazo durante esta etapa es debido a que se caracteriza por la baja cantidad de controles prenatales, los cuales no suelen ser planeados y se corre el riesgo de repetir un embarazo en esta etapa. Asimismo, se han reportado resultados como bajo peso al nacer, parto prematuro, muerte fetal, preeclampsia, hemorragia post parto y endometritis puerperal asociados al embarazo adolescente. ${ }^{4-8}$

Por otro lado, estudios reportan cómo las creencias locales pueden ser determinantes del embarazo adolescente; en donde, por ejemplo, el sexo prematrimonial llega a ser una norma de ciertos entornos sociales, asimismo, el bajo conocimiento suele ser una constante de los embarazos durante esta etapa. ${ }^{9}$ Estos estudios que evidencian los factores que predisponen el embarazo adolescente permiten brindar un marco con el cual diseñar los servicios especializados para adolescentes. ${ }^{10}$

Es así como la presente investigación busca brindar un aporte en el conocimiento de ciertos determinantes del embarazo adolescente en el primer nivel de atención, el cual según la normativa peruana es la puerta de ingreso al sistema de salud y donde aún existe poca evidencia respecto al tema; esperando que pueda ser de utilidad en el diseño de los servicios diferenciados que se vienen implementando. "Por lo cual, el objetivo del presente estudio es determinar los factores asociados al embarazo adolescente en un centro materno infantil de Lima durante el 2018.

\section{MATERIAL Y MÉTODOS}

Estudio analítico de casos y controles donde participaron adolescentes (10 a 19 años) ${ }^{12}$ atendidas en el Centro de
Salud Materno Infantil "Santa Anita", establecimiento I-4 del Ministerio de Salud ubicado en Lima (Perú), durante mayo a julio del 2018.

Para el cálculo del tamaño de la muestra se utilizó el software libre OpenEpi (http://www.openepi.com/Menu/OE_Menu.htm) mediante la fórmula para estudio de casos y controles no pareados. Se consideró un nivel de confianza de $95 \%$, una potencia del $80 \%$, una razón de controles por caso de 2 . Asimismo, la proporción de controles expuestos fue del $56.5 \%$ y el Odds Ratio de 12.5 , según un estudio previo. ${ }^{13}$ Con lo cual se obtuvo un tamaño de muestra de 57 participantes, distribuidos en 19 casos y 38 controles. Se utilizó un muestreo probabilístico de tipo aleatorio simple.

Se consideraron como casos a las adolescentes que acudían al servicio de obstetricia por su control prenatal, lo cual fue corroborado mediante el carné perinatal, mientras que el grupo control estuvo conformado por aquellas adolescentes que acudan al establecimiento por algún motivo ajeno a un embarazo o como acompañantes de algún familiar. Se excluyeron a quienes hayan presentado un embarazo anteriormente $y / 0$ muestren algunas discapacidad mental. Finalmente, se eliminaron los registros de participantes que no completaron adecuadamente el instrumento de investigación.

La variable dependiente del estudio fue el embarazo adolescente, categorizado como "presenta" (casos) y "no presenta" (controles). La variable independiente estuvo conformada por los factores asociados, dimensionados en factores individuales (estado civil, grado de instrucción, ocupación, uso de métodos anticonceptivos, edad, edad de la primera menstruación, edad de la primera relación sexual y número de parejas sexuales), familiares (fallecimiento del familiar, estado civil de los padres, grado de instrucción de los padres y presencia de familiar con embarazo adolescente) y sociales (ingreso económico, número de personas que viven en el hogar y presencia de servicios básicos).

La técnica utilizada fue la encuesta personalizada a través de preguntas incluidas en un cuestionario autoaplicable, el cual fue entregado luego de que acepte formalmente participar en el estudio. La recolección de datos se realizó en el ambiente de espera de los consultorios del establecimiento. Los factores incluidos como posibles determinantes del embarazo adolescente fueron obtenidos de la revisión de estudios previos. 
El análisis se realizó mediante el software STATA. Las variables categóricas fueron reportadas mediante frecuencias y porcentajes, mientras que las numéricas se reportaron mediante medias y desviación estándar. La variación significativa de promedios fue evaluada mediante T de Student, debido a la distribución normal de las variables numéricas. La evaluación de los factores asociados fue realizada mediante la prueba Chi Cuadrado de Pearson, considerando un nivel de confianza del $95 \%$ y asumiendo como factor asociado a todo $\mathrm{p}$ valor menor a 0.05 .

El estudio fue revisado y aprobado inicialmente por el comité de investigación de la Escuela Profesional de Obstetricia de la Universidad Nacional Mayor de San Marcos, para luego solicitar los permisos en el establecimiento sanitario. Se consideró en todo momento la voluntariedad de la participación, la cual fue confirmada mediante la firma de un consentimiento o asentimiento informado, dependiendo de la edad del participante, tomando en cuenta la opinión del padre con quien acudía. Se mantuvo la confidencialidad de los participantes mediante la codificación de los datos identificables.

\section{RESULTADOS}

En el estudio participaron 57 adolescentes, divididas en 38 gestantes (casos) y 19 no gestantes (controles). Se generó un análisis para ver los diversos factores asociados donde la primera dimensión fueron los factores individuales. En la Tabla 1 se muestra que el estado civil fue un factor asociado ( $p<0.001)$, donde un $73.68 \%(n=14)$ de las gestantes adolescentes presentaban un grado de instrucción conviviente, a diferencia de las no gestantes que eran todas solteras. Respecto al grado de instrucción $(p=0.005)$, la mayor proporción presentó secundaria completa. La ocupación $(p=0.002)$ fue de ama de casa en mayor proporción en adolescentes gestantes $(42.11 \%)$, a diferencia de las no gestantes, donde la principal ocupación fue de estudiantes $(71.05 \%)$. Finalmente, el uso de métodos anticonceptivos $(p=0.008)$ fue mayor en gestantes adolescentes (52.63\%) que en el grupo controles (18.42\%).

Entre los factores individuales numéricos, se halló que la edad promedio de las gestantes adolescentes fue de 17.89 años, mientras que las no gestantes fue de 16.39 años.

Tabla 1. Factores individuales asociados al embarazo adolescente

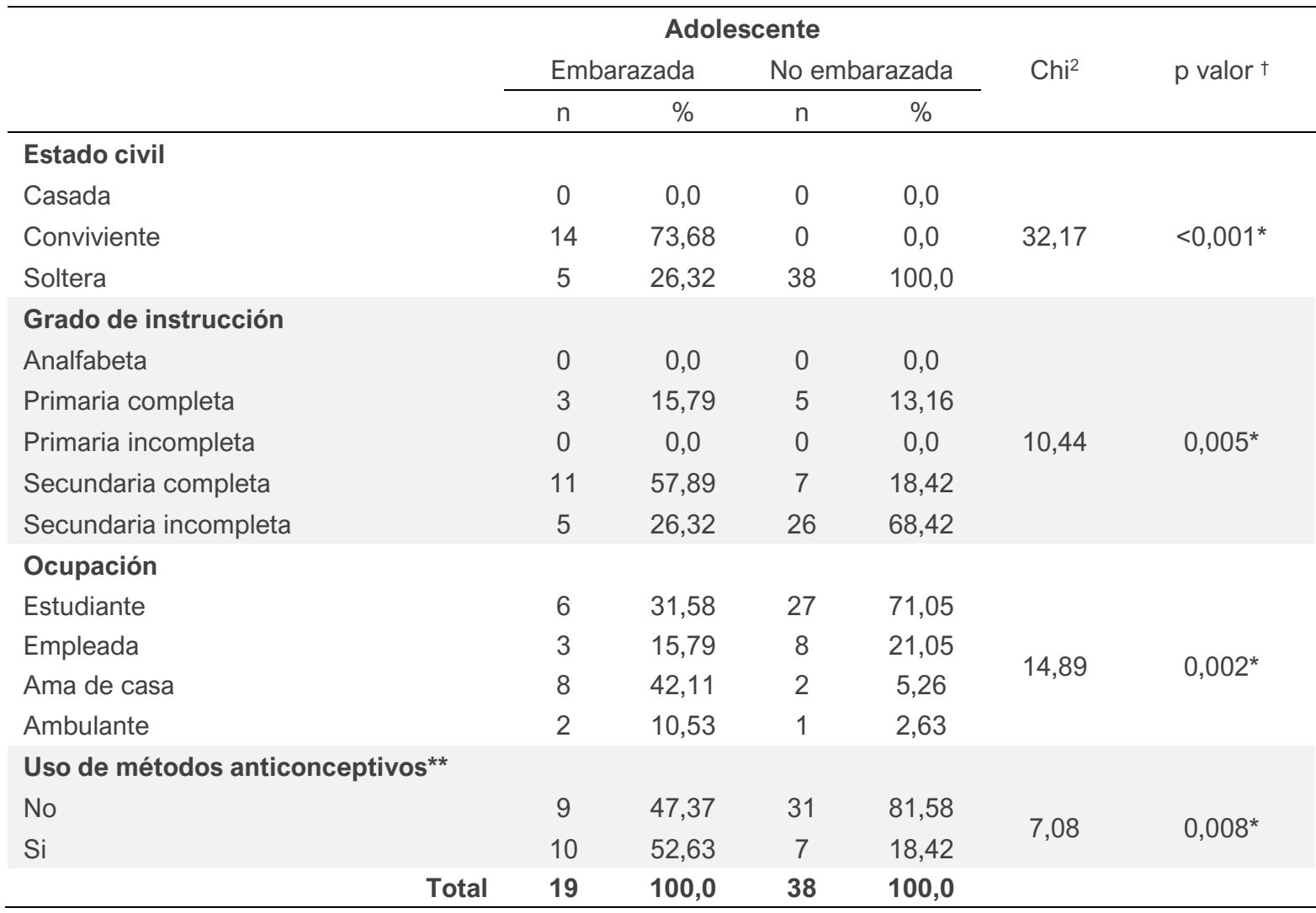

† Evaluado mediante la prueba Chi Cuadrado de Pearson

* Factores asociados $(p<0,05)$

** Uso actual, al momento del estudio 
Tabla 2. Factores individuales relacionados al embarazo adolescente

\begin{tabular}{lcccccc}
\hline & \multicolumn{5}{c}{ Adolescente } & \\
& \multicolumn{2}{c}{ Embarazada } & No embarazada & \multirow{2}{*}{ p valor ${ }^{\dagger}$} \\
\cline { 2 - 5 } & Media & D.S & Media & D.S & \\
\hline Edad & 17,89 & 1,24 & 16,39 & 1,33 & $<0,001$ \\
Edad de la primera menstruación & 13,00 & 1,37 & 12,39 & 1,39 & 0,125 \\
Edad de su primera relación sexual & 16,37 & 1,34 & 15,42 & 0,79 & 0,039 \\
Número de parejas sexuales & 1,95 & 1,22 & 0,45 & 0,76 & $<0,001$ \\
\hline
\end{tabular}

† Evaluado mediante la prueba $\mathrm{T}$ de Student para varianzas iguales

Edad reportada en años

D.S: Desviación estándar

La edad de la primera menstruación y de la primera relación sexual también resultó mayor en el grupo de gestantes adolescentes. El número de parejas sexuales promedio fue de $1.95 \pm 1.22$ en adolescentes que se encuentran gestando y de $0.45 \pm 0.76$ en adolescentes que no han gestado hasta el momento. (Tabla 2)
Entre los factores familiares, en la Tabla 3 se muestra que el grado de instrucción de los padres $(p=0.002)$ de las gestantes adolescentes fue principalmente de secundaria incompleta (36.84\%), mientras que las que no estuvieron gestando presentaron padres con grado de instrucción de secundaria completa (65.79\%).

Tabla 3. Factores familiares asociados al embarazo adolescente

\begin{tabular}{|c|c|c|c|c|c|c|}
\hline & \multicolumn{4}{|c|}{ Adolescente } & \multirow{3}{*}{$\mathrm{Chi}^{2}$} & \multirow{3}{*}{$p$ valor ${ }^{\dagger}$} \\
\hline & \multicolumn{2}{|c|}{ Embarazada } & \multicolumn{2}{|c|}{ No embarazada } & & \\
\hline & $\mathrm{n}$ & $\%$ & $\mathrm{n}$ & $\%$ & & \\
\hline \multicolumn{7}{|l|}{ Fallecimiento de familiar } \\
\hline Padre & 3 & 15,79 & 1 & 2,63 & \multirow{3}{*}{3,77} & \multirow{3}{*}{0,151} \\
\hline Madre & 0 & 0,0 & 1 & 2,63 & & \\
\hline Ninguno & 16 & 84,21 & 36 & 94,74 & & \\
\hline \multicolumn{7}{|l|}{ Estado civil de los padres } \\
\hline Casados & 4 & 21,05 & 12 & 31,58 & \multirow{3}{*}{1.78} & \multirow{3}{*}{0.411} \\
\hline Convivientes & 7 & 36,84 & 8 & 21,05 & & \\
\hline Divorciados/Separados & 8 & 42,11 & 18 & 47,37 & & \\
\hline \multicolumn{7}{|l|}{ Grado de instrucción de los padres } \\
\hline Analfabeta & 2 & 10,53 & 0 & 0,0 & \multirow{5}{*}{17,42} & \multirow{5}{*}{$0,002^{*}$} \\
\hline Primaria completa & 3 & 15,79 & 2 & 5,26 & & \\
\hline Primaria incompleta & 4 & 21,05 & 1 & 2,63 & & \\
\hline Secundaria completa & 3 & 15,79 & 25 & 65,79 & & \\
\hline Secundaria incompleta & 7 & 36,84 & 10 & 26,32 & & \\
\hline \multicolumn{7}{|l|}{$\begin{array}{l}\text { Miembro familiar presentó embarazo } \\
\text { durante la adolescencia }\end{array}$} \\
\hline Ninguno & 6 & 31,58 & 17 & 44,74 & \multirow{6}{*}{13.24} & \multirow{6}{*}{$0.021^{*}$} \\
\hline Abuela & 0 & 0,0 & 1 & 2,63 & & \\
\hline Mamá & 9 & 47,37 & 5 & 13,16 & & \\
\hline Hermana & 0 & 0,0 & 6 & 15,79 & & \\
\hline Primas & 1 & 5,26 & 7 & 18,42 & & \\
\hline Tía & 3 & 15,79 & 2 & 5,26 & & \\
\hline Total & 19 & 100,0 & 38 & 100,0 & & \\
\hline
\end{tabular}

† Evaluado mediante la prueba Chi Cuadrado de Pearson

* Factores asociados $(p<0,05)$ 
Tabla 4. Factores sociales asociados al embarazo adolescente

\begin{tabular}{|c|c|c|c|c|c|c|}
\hline & \multicolumn{4}{|c|}{ Adolescente } & \multirow{3}{*}{$\mathrm{Chi}^{2}$} & \multirow{3}{*}{$\mathrm{p}$ valor ${ }^{\dagger}$} \\
\hline & \multicolumn{2}{|c|}{ Embarazada } & \multicolumn{2}{|c|}{ No embarazada } & & \\
\hline & $\mathrm{n}$ & $\%$ & $\mathrm{n}$ & $\%$ & & \\
\hline \multicolumn{7}{|l|}{ Ingreso económico familiar mensual } \\
\hline Menos de S/.100 & 0 & 0,0 & 4 & 10,53 & \multirow{4}{*}{3,90} & \multirow{4}{*}{0,272} \\
\hline De $S /, 100$ a $S / .500$ & 15 & 78,95 & 21 & 55,26 & & \\
\hline De S/.600 a S/.1000 & 3 & 15,79 & 9 & 23,68 & & \\
\hline De S/.1000 a más & 1 & 5,26 & 4 & 10,53 & & \\
\hline \multicolumn{7}{|l|}{ Personas que viven en el hogar } \\
\hline 1 a 2 & 6 & 31,58 & 3 & 7,89 & \multirow{4}{*}{10,87} & \multirow{4}{*}{$0,012^{*}$} \\
\hline 3 a 4 & 6 & 31,58 & 5 & 13,16 & & \\
\hline 5 a 6 & 3 & 15,79 & 19 & 50,00 & & \\
\hline 7 a más & 4 & 21,05 & 11 & 28,95 & & \\
\hline \multicolumn{7}{|l|}{ El hogar cuenta con servicios básicos } \\
\hline $\mathrm{Si}$ & 19 & 100.0 & 38 & 100.0 & \multirow{3}{*}{$-* *$} & \multirow{3}{*}{$-* *$} \\
\hline No & 0 & 0.0 & 0 & 0.0 & & \\
\hline Total & 19 & 100,0 & 38 & 100,0 & & \\
\hline
\end{tabular}

Asimismo, el tener un familiar que haya presentado un embarazo durante la adolescencia también se mostró como un factor asociado $(p=0.021)$, en donde el $47.37 \%$ del grupo de gestantes adolescente tuvo una madre que también tuvo un embarazo adolescente, mientras que en el grupo control el $44.74 \%$ no tuvo ningún familiar con un embarazo adolescente previo.

Finalmente, entre los factores sociales, el número de personas que viven en el hogar se mostró como un factor asociado $(p=0.012)$, en donde el grupo de gestantes adolescentes mostró mayor proporción de 1 a 2 personas que viven en el hogar (31.58\%), mientras que en el grupo control la mayor proporción (50\%) tenía entre 5 a 6 familiares viviendo en su hogar. El ingreso económico familiar no se halló como un factor asociado y todas las participantes refirieron que su hogar cuenta con servicios básicos. (Tabla 4)

\section{DISCUSIÓN}

Entre los factores individuales, el grado de instrucción se asoció significativamente con la presencia del embarazo durante la adolescencia, tal como se reporta en estudios previos donde se encontró que el presentar un mayor nivel educativo se comporta como un factor protector del embarazo adolescente ${ }^{14}$, del mismo modo la ausencia de educación incrementa en 3 veces la probabilidad de un embarazo en esta etapa de la vida. ${ }^{15,16}$ Por otro lado, la ocupación mostró que el embarazo adolescente puede llegar a generar deserción escolar para ocupar el tiempo en trabajar o ser ama de casa; lo cual coincide con reportes del Hospital San Juan de Lurigancho ${ }^{17}$ donde la deserción se da en el $43 \%$ de las adolescentes gestantes. Al respecto otros investigadores añaden que este abandono escolar genera una serie de eventos que establecen la marginación social, subdesarrollo y pobreza. ${ }^{18}$

El uso de métodos anticonceptivos presentó una característica peculiar, y es que gran parte de los que referían utilizarlos fueron las gestantes adolescentes, cuando en diversas investigaciones ${ }^{19}$ se ha encontrado que es justamente este grupo quien presenta mayor proporción de no uso de métodos anticonceptivos. Esto puede deberse a que la pregunta estuvo enfocada al uso actual de métodos, y el hecho de que una adolescente esté gestando puede haber modificado su conducta hacia el uso de métodos.

Respecto a la menarquia, un estudio realizado en Lima 20 mostró que el presentar la primera menstruación a una menor edad llegaba a generar en la adolescente un riesgo de 2.1 veces de presentar un embarazo; sin embargo, esta sentencia es refutada con el presente estudio. 
Asimismo, la edad de la primera relación sexual, variable que resultó asociada y que mostró que el inicio de relaciones sexuales suele ser más tardía en gestantes adolescentes, no coincide con estudios previos ${ }^{19,21}$ donde reportan que la edad de inicio sexual en gestantes adolescentes suele ser antes de los 16 años.

Dentro de los factores familiares, una de las variables evaluadas fue la presencia de un miembro que haya tenido un embarazo durante su adolescencia, la cual llegó a ser un factor asociado, dando a entender que el modelo de un pariente cercano puede influir en un embarazo adolescente actual. Al respecto existen diversas investigaciones que apoyan este resultado ${ }^{15}$, donde se menciona que el tener hermanos sexualmente activos o que han quedado embarazadas a una edad temprana se muestran como factores de riesgo; asimismo, en Colombia ${ }^{19}$ se encontró que el tener amigas que han presentado un embarazo durante la adolescencia, independientemente si lo abortaron o no, llega a ser un factor asociado para un presente embarazo adolescente.

Otro factor familiar asociado fue el grado de instrucción de los padres, donde las adolescentes que no están gestando suelen tener padres con un mayor nivel educativo. Al respecto, en Colombia ${ }^{16}$ un estudio determinó que tener una madre con educación menor a la secundaria se comporta como un factor de riesgo para la presencia de embarazo adolescente. Asimismo, estudios en este país ${ }^{13,16}$ reportan que el estrato económico también muestra asociación con el embarazo adolescente, lo cual no coincide con esta investigación.

Por otro lado, un factor social asociado al embarazo adolescente fue el número de personas que conviven con la adolescente en el hogar; notándose nuevamente el impacto que pueden tener los familiares en la presencia de un embarazo a esta edad. Al respecto, evidencia encontró que existen actitudes negligentes por parte de los padres o familiares que pueden favorecer al embarazo adolescente ${ }^{22}$, tales como descuido en el afecto o el acompañamiento, en la ayuda de sus tareas, instauración de límites o educación sobre la sexualidad, entre otros.

Cabe mencionar que, entre las limitaciones que pudieron retrasar los tiempos estimados y podrían tomarse en cuenta para estudios posteriores, se halló que las gestantes adolescentes no suelen acudir a su control prenatal correspondiente, por lo cual hubo situaciones en las que se acompañó al profesional obstetra en sus visitar domiciliarias, para así aprovechar en entrevistar a las gestantes que no acudían a su control programado. Otra limitación del estudio fue el permiso por parte de los padres de las adolescentes para participar en el estudio.

Finalmente, se concluye que los factores individuales que se asocian al embarazo adolescente son el estado civil, grado de instrucción, ocupación, uso de métodos anticonceptivos, edad del adolescente, edad de su primera relación sexual y andría. Entre los factores familiares se asocian el grado de instrucción de los padres y el antecedente de algún familiar cercano con embarazo adolescente. Entre los factores asociales el número de personas que viven en el hogar se asocian al embarazo adolescente. 


\section{REFERENCIAS BIBLIOGRÁFICAS}

1. Caffe S Caffe S, Plesons M, Camacho AV, Brumana L, Abdool SN, Huaynoca S. Looking back and moving forward: can we accelerate progress on adolescent pregnancy in the Americas? Reprod Health. 14 de julio de 2017;14(1):83. [Link]

2. Holness N. A global perspective on adolescent pregnancy. Int J Nurs Pract. octubre de 2015;21(5):677-81. [Link]

3. Ministerio de Salud del Perú. Embarazo adolescente en 2017 llegó al $13.4 \%$ [Internet]. [citado 19 de abril de 2019]. [Link]

4. Leftwich HK, Alves MVO. Adolescent Pregnancy. Pediatr Clin North Am. 2017;64(2):381-8. [Link]

5. Conde-Agudelo A, Belizán JM, Lammers C. Maternal-perinatal morbidity and mortality associated with adolescent pregnancy in Latin America: Cross-sectional study. Am J Obstet Gynecol. febrero de 2005;192(2):342-9. [Link]

6. Ganchimeg T, Mori R, Ota E, Koyanagi A, Gilmour S, Shibuya K. Maternal and perinatal outcomes among nulliparous adolescents in low- and middleincome countries: a multi-country study. BJOG Int J Obstet Gynaecol. diciembre de 2013;120(13):1622-30 discussion 1630. [Link]

7. Ganchimeg T, Ota E, Morisaki N, Laopaiboon M, Lumbiganon P, Zhang J. Pregnancy and childbirth outcomes among adolescent mothers: a World Health Organization multicountry study. BJOG Int J Obstet Gynaecol. marzo de 2014;121 Suppl 1:40-8. [Link]

8. De Azevedo WF, Diniz MB, da Fonseca ESVB, de Azevedo LMR, Evangelista CB. Complications in adolescent pregnancy: systematic review of the literature. Einstein. 2015;13(4):618-26. [Link]
9. Sychareun V, Vongxay V, Houaboun S, Thammavongsa $V$, Phummavongsa $P$, Chaleunvong $\mathrm{K}$, et al. Determinants of adolescent pregnancy and access to reproductive and sexual health services for married and unmarried adolescents in rural Lao PDR: a qualitative study. BMC Pregnancy Childbirth [Internet]. 8 de junio de 2018 [citado 20 de abril de 2019];18. [Link]

10. Estrada F, Suárez-López L, Hubert C, Allen-Leigh B, Campero L, CruzJimenez L. Factors associated with pregnancy desire among adolescent women in five Latin American countries: a multilevel analysis. BJOG Int J Obstet Gynaecol. septiembre de 2018;125(10):1330-6. [Link]

11. Ministerio de Salud del Perú. Adolescentes reciben atención diferenciada en establecimientos de salud a nivel nacional [Internet]. [citado 20 de abril de 2019]. [Link]

12. Organización Mundial de la Salud. Desarrollo en la adolescencia [Internet]. WHO. [citado 16 de abril de 2019]. [Link]

13. Gómez-Mercado CA, Montoya-Vélez LP. Factores sociales, demográficos, familiares y económicos relacionados con el embarazo en adolescentes, área urbana, Briceño, 2012. Rev Salud Pública. 2014;16(3):394-406. [Link]

14. Degollar A, Antoinette V. Factores individuales, familiares y sociales asociados al embarazo en adolescentes, Centro Materno Infantil José Gálvez, Lima - Perú, 2014. Repos Tesis - UNMSM [Internet]. 2016 [citado 19 de abril de 2019]. [Link]

15. Akanbi F, Afolabi KK, Aremu AB. Individual Risk Factors Contributing to the Prevalence of Teenage Pregnancy among Teenagers at Naguru Teenage Centre Kampala, Uganda. Prim Health Care Open Access [Internet]. 2016 [citado 19 de abril de 2019]; 06 (04). [Link]
16. Sánchez-Valencia YA, MendozaTascón LA, Grisales-López MB, Ceballos-Molina LY, BustamenteFarfan JC, Castañeda EM. Características poblacionales y factores asociados a embarazo en mujeres adolescentes de Tuluá, Colombia. Rev Chil Obstet Ginecol. 2013;78(4):269-81. [Link]

17. López-Untiveros LN, Luna-Vásquez SS. Factores asociados al embarazo adolescente en las gestantes que acuden al Hospital San Juan de Lurigancho. Repos Inst - Wien [Internet]. 2014 [citado 19 de abril de 2019]. [Link]

18. Ulloque-Caamaño L, MonterrosaCastro Á, Arteta-Acosta C. Prevalencia de baja autoestima y nivel de resiliencia bajo, en gestantes adolescentes de poblaciones del caribe colombiano. Rev Chil Obstet Ginecol. diciembre de 2015;80(6):46274. [Link]

19. Carvajal Barona R, Valencia Oliveros $\mathrm{HL}$, Rodríguez Amaya RM. Factores asociados al embarazo en adolescentes de 13 a 19 años en el municipio de Buenaventura, Colombia. Rev Univ Ind Santander Salud [Internet]. 2017 [citado 19 de abril de 2019];49(2). [Link]

20. Marcellini L, Fiorella D. Factores individuales, familiares y sociales asociados al embarazo en adolescentes en el Centro de Salud Carlos Showing Ferrari 2014 - 2015. Univ Huánuco [Internet]. 2017 [citado 19 de abril de 2019]. [Link]

21. Osorio Alania R. Factores que se asocian al embarazo en adolescentes atendidas en el Hospital de Apoyo María Auxiliadora, 2013. Univ Nac Mayor San Marcos [Internet]. 2014 [citado 19 de abril de 2019]. [Link]

22. Salazar A, Navarrete VCÁ, Velásquez CB. Negligencia del padre: Una práctica que puede llevar al embarazo adolescente. Rev Colomb Cienc Soc. 2018;9(1):51-75. [Link] 